\title{
¿Por qué y para qué se investiga en Ciencias Sociales? Concepciones del personal investi- gador en formación
}

\section{Manuela Alvarez Alvarez ${ }^{1}$, Iciar Elexpuru ${ }^{2}$, Montserrat Castelló $^{3}$, Lourdes Villardón-Gallego ${ }^{4}$, Concepción Yániz ${ }^{5}$.}

\author{
${ }^{1}$ Facultad de Ciencias Sociales y Humanas, Universidad de Deusto, Bilbao \\ 2, 4 y 5 Facultad de Psicología y Educación, Universidad de Deusto, Bilbao \\ ${ }^{3}$ Facultad de Psicología, Ciencias de la Educación y del Deporte, FPCEE. Blan- \\ querna. Universitat Ramon Llull, Barcelona
}

\section{España}




\section{Resumen}

Introducción. El conocimiento sobre lo que significa investigar para los investigadores en formación en el contexto español es prácticamente inexistente. La finalidad de este estudio es analizar las concepciones sobre la investigación de los estudiantes de doctorado en ciencias sociales y la relación de estas concepciones con algunas variables relevantes en el proceso de formación investigadora.

Método. Participaron 1082 estudiantes de 56 universidades españolas que respondieron a un cuestionario de 33 ítems tipo Likert con siete opciones de respuesta según grado de acuerdo, sobre concepciones, compromiso e intereses por la investigación; relación con la comunidad científica y estrés. Se adoptó un diseño observacional de tipo transversal. Se realizaron análisis descriptivos de tendencia central y dispersión, ANOVA y r de Pearson (SPSS, v.23).

Resultados. Los doctorandos entienden la investigación prioritariamente como un proceso orientado tanto a ofrecer una contribución a la comunidad como al desarrollo personal, y con menor frecuencia, como un producto que se plasma en la publicación de artículos.

Discusión y conclusiones. Este estudio permite situar las concepciones en las categorías orientación de la investigación hacia el desarrollo individual o de la comunidad y hacia el proceso o producto, dimensiones coherentes con la investigación previa en el ámbito internacional. Los resultados tienen implicaciones para el diseño de propuestas formativas en el doctorado que consideren tanto la comprensión de lo que significa investigar como las expectativas laborales de los estudiantes.

Palabras Clave: concepciones sobre investigación, identidad del investigador, investigadores en formación, doctorado, investigación en ciencias sociales. 


\begin{abstract}
Introduction. There is virtually no knowledge regarding what research means for researchers in training in the Spanish context. This study has a twofold goal: to analyze the conceptions of $\mathrm{PhD}$ students in the Social Sciences regarding research, and the relations of those conceptions with several relevant variables in the process of researcher training.

Method. 1082 students from 56 Spanish universities participated in this study. They answered a 33-items questionnaire Likert-type with seven answers according level of agreement on conceptions, commitment, interest for research, relationship with the scientific community, and stress. An observational transversal design was adopted. Descriptive analyzes of central tendency and dispersion, ANOVA, and r of Pearson (SPSS, v.23) were performed.
\end{abstract}

Results. PhD students conceptualize research mainly as a process oriented activity both towards making a contribution to the community and towards personal development; additionally, albeit less often, they conceptualize it as a product: papers published for others to read.

Discussion and Conclusion. This study situates these conceptions into categories accounting for the research as being more individual or community oriented; more process or product oriented. There is a certain agreement on these categories in previous research in the international context. The results of this study have implications for the design of training proposals for doctoral education that take into account the understanding of what research means, together with students' job expectations.

Keywords: conceptions on research, researcher identity, student research, doctoral degrees, social science research. 


\section{Introducción}

El contexto cambiante, complejo y altamente competitivo de la Educación Superior ha generado interés por estudiar los procesos educativos que tienen lugar en las universidades. Este interés se asocia especialmente a la formación de investigadores en los estudios de doctorado y, en particular, en los últimos años, a la necesidad de avanzar en la comprensión de concepciones sobre la investigación. Mientras que son numerosas las investigaciones sobre lo que significa enseñar y ser un docente universitario, los estudios sobre lo que significa investigar y ser un investigador son escasos (Akerlind, 2008).

En el ámbito español, la mayoría de estos estudios se ha ocupado de evaluar el nivel de productividad científica de los doctorados o las relaciones de la productividad con otras variables como la normativa, las áreas de conocimiento o la financiación (Buela-Casal, Bermudez, Sierra, Ramiro y Castro, 2011; Musi-Lechuga, Olivas-Avila y Castro, 2011). A pesar de la relevancia y utilidad de estas iniciativas, son prácticamente inexistentes los estudios que abordan la investigación desde la perspectiva de los protagonistas, los retos e incidentes que experimentan y sus necesidades de formación (Castelló, Iñesta y Corcelles, 2013). Sin embargo, en EEUU, Australia y Canadá son habituales los estudios que evalúan la experiencia de los investigadores noveles durante sus estudios de doctorado y en el periodo postdoctoral (Mcalpine, Jazvac-Martek y Hopwood, 2009; McAlpine y McKinnon, 2012; McAlpine, Amundsen y Turner, 2013; 2014). También en el contexto europeo hay estudios sobre aspectos generales y específicos de la experiencia del doctorado. Destacan los trabajos sobre las concepciones que los estudiantes desarrollan en contextos específicos (Martinsuo y Turkulainen, 2011; Mainhard, van der Rijst, van Tartwijk y Wubbels, 2009) y los que analizan estas concepciones desde perspectivas transculturales (Donahue, 2008; Chitez y Kruse, 2012).

Mención aparte merecen los trabajos de Pyhältö y Lonka, en los que se establecen relaciones entre diferentes aspectos clave de los estudios de doctorado, como por ejemplo, bienestar, compromiso, inserción en la comunidad de referencia o escritura (Pyhältö, Stubb y Lonka, 2009; Stubb, Pyhältö y Lonka, 2012). Estos estudios muestran cómo las concepciones sobre la investigación son variables predictoras de la finalización de la tesis doctoral y del desarrollo profesional como investigadores, resultados avalados por algunos estudios españo- 
les (Castelló, Iñesta y Monereo, 2009; Castelló, González e Iñesta, 2010), que ponen de manifiesto la importancia de conocer y caracterizar las concepciones de los investigadores noveles. Los académicos están habituados a plantear su propia investigación y a justificar sus resultados, pero no tanto a elaborar lo que significa investigar en sí (Stubb, Phylalto y Lonka, 2014).

El interés por estudiar las concepciones sobre la investigación es bastante reciente y la mayor parte de la literatura se ha publicado durante la última década. Akerlind (2008) identificaba sólo diez estudios posteriores al trabajo pionero de Brew (2001); además, la mayoría de los estudios se centra en las concepciones de los investigadores senior (Meyer, Shanahan y Laugksch, 2005, 2007). Sólo en los últimos años se han desarrollado algunos estudios sobre los investigadores noveles (Pitcher, 2010; 2011; Stubb et al., 2014) cuyos resultados coinciden en señalar algunas dimensiones y categorías útiles para explicar las concepciones sobre la investigación. Así, las concepciones se sitúan a lo largo de dos dimensiones relativas a la orientación del investigador hacia lo individual o la comunidad, por un lado y hacia el proceso o el producto, por otro (Stubb et al., 2014). Basándose en estas dimensiones, hay cierto acuerdo en el establecimiento, naturaleza y alcance de categorías que corresponden a diferentes maneras de percibir la investigación (Akerlind, 2008; Pitcher y Akerlind, 2009, Pitcher, 2009; Stubb et al., 2014). Akerlind (2008) distingue entre intenciones de investigación, preguntas, proceso y resultados. De modo similar, pero centrándose exclusivamente en las concepciones de los estudiantes de doctorado, Stubb et al. (2014) analizan específicamente las razones o las metas para investigar y las formas en que se logran dichas metas.

En cuanto a la metodología, prácticamente toda la investigación realizada hasta el momento ha sido de carácter cualitativo, basada en entrevistas o cuestionarios con preguntas abiertas. Algunos estudios han utilizado metáforas como herramienta para acceder a las concepciones (Visser-Wijnveen, Van Driel, Van der Rijst, Verloop y Visser, 2009; Pitcher, 2011). Meyer et al. (2005, 2007) utilizaron un enfoque metodológico diferente al desarrollar el Students' Conceptions of Research Inventory (SCoRI), para validar empírica y psicométricamente las dimensiones relativas a las concepciones sobre la investigación de los estudiantes. Los ítems del SCoRI proceden de un estudio cualitativo previo en el que se establecieron varias categorías preliminares. Los factores finales son conceptualmente coherentes con los resultados de la investigación previa y pueden explicarse en términos de las dimensiones mencionadas anteriormente: orientación de la investigación centrada en el desarrollo indivi- 
dual o de la comunidad y percepción de la investigación como fundamentalmente orientada al proceso o al producto.

Este cuestionario tiene algunas limitaciones que dificultan su uso. Primero, la excesiva extensión de la versión final (69 ítems) lo que puede ser un obstáculo para mantener el compromiso de respuesta hasta su finalización. Segundo, el hecho de que no todos los factores y categorías cualitativas obtuvieron el mismo soporte empírico. A pesar de que todos los estudios revisados enfatizan la necesidad de ahondar en las concepciones sobre la investigación para ayudar a los estudiantes a desarrollar una adecuada trayectoria doctoral, el conocimiento actual sobre los motivos que llevan a los estudiantes a implicarse en el proceso doctoral y a perseverar ante los retos que dicho proceso plantea es todavía fragmentado y escaso (Vekkalia, Pyhältö, \& Lonka, 2013; Villardón-Gallego y Yániz, 2013).

\section{Objetivos}

Esta investigación pretende contribuir al aumento de este conocimiento y para ello se plantea los siguientes objetivos: 1) conocer las concepciones de los doctorandos españoles de CCSS sobre lo que significa investigar; 2) analizar la relación existente entre dichas concepciones y diferentes variables académicas y sociodemográficas; y 3) estudiar qué vinculación tienen las distintas concepciones con el compromiso y tipo de interés en la investigación, la integración en la comunidad científica, así como el estrés experimentado en el proceso.

\section{Método}

\section{Participantes}

La muestra estuvo formada por 1082 estudiantes de doctorado de CCSS: Psicología (34.7\%), Educación (19.3\%), Economía (18.9\%), Derecho (12.3\%), Ciencias Políticas y de la Información (14.8\%); de 56 universidades españolas (74\% de la población); 59.7\% hombres y $40.3 \%$ mujeres. La media de edad de los participantes fue 36.85 (DT = 9.2), 21.1\% menores de 30 años, $32.3 \%$ entre 30 y 39, $16.1 \%$ entre 40 y 49 y 9.3\% 50 o más años.

\section{Instrumentos}

Se utilizó el cuestionario Formación de la Identidad del Investigador Novel en CCSS, diseñado en formato online a través de la aplicación Lime-Survey. Para este estudio se anali- 
zaron las siguientes escalas (tipo Likert con siete opciones de respuesta, desde 1 "totalmente en desacuerdo" hasta 7 "completamente de acuerdo"): concepciones sobre la investigación, compromiso e intereses por la investigación; relación con la comunidad científica y estrés).

La escala de concepciones sobre la investigación se diseñó a partir del SCoRI (Meyer et al. 2005, 2007), y recoge aquellos ítems refrendados por investigaciones posteriores (Stubbs et al. 2014) lo que garantiza la validez de constructo. Se eligieron ocho ítems siguiendo criterios de a) fiabilidad (en términos de su peso factorial) y b) representatividad de las diferentes categorías (ver Tabla1). Los resultados confirman la fiabilidad de la escala con un alpha de Cronbach de .751 .

Tabla1. Concepciones sobre qué es investigar según el continuo orientación al individuo o a la comunidad y orientación al proceso o al producto

\begin{tabular}{|c|c|c|}
\hline & Orientado al Proceso & Orientado al Producto \\
\hline \multirow[t]{2}{*}{$\begin{array}{l}\text { Orientado al indi- } \\
\text { viduo }\end{array}$} & $\begin{array}{l}\text { 5. Investigar es una cuestión de } \\
\text { desarrollo personal }\end{array}$ & $\begin{array}{l}\text { 8. Investigar tiene que ver } \\
\text { con ser influyente o reco- } \\
\text { nocido/a en tu área de }\end{array}$ \\
\hline & $\begin{array}{l}\text { 2. Investigar es una especie de } \\
\text { lucha, en la que hay que deci- } \\
\text { dir un tema lo suficientemente } \\
\text { interesante para perseverar en } \\
\text { su estudio }\end{array}$ & $\begin{array}{l}\text { 1. Investigar tiene que ver } \\
\text { con el hecho de publicar } \\
\text { artículos y que otros los } \\
\text { lean }\end{array}$ \\
\hline \multirow[t]{2}{*}{$\begin{array}{l}\text { Orientado a la co- } \\
\text { munidad }\end{array}$} & $\begin{array}{l}\text { 3. Investigar tiene que ver so- } \\
\text { bre todo con comparar; por } \\
\text { ejemplo, se comparan resulta- } \\
\text { dos nuevos con los previos }\end{array}$ & $\begin{array}{l}\text { 6. Investigar tiene que ver } \\
\text { con completar lo que em- } \\
\text { pezaste }\end{array}$ \\
\hline & $\begin{array}{l}\text { 7. Investigar es relevante no } \\
\text { solamente para uno mismo sino } \\
\text { también porque los resultados } \\
\text { pueden beneficiar a otras }\end{array}$ & $\begin{array}{l}\text { 4. Investigar tiene que ver } \\
\text { con juntar diferentes piezas } \\
\text { de información, sintetizar- } \\
\text { las u organizarlas de forma }\end{array}$ \\
\hline
\end{tabular}


sentido

El resto de las escalas (ver Tabla 2) se adaptaron de instrumentos previos ya validados (Pyhältö, Vekkaila y Keskinen, 2015; Pyhältö, Peltonen, Rautio, Haverinen, Laatikainen y Vekkaila, 2016; Castelló, McAlpine, \& Pyhältö, 2017; Castelló, Pardo, Sala-Bubaré, \& Suñe, 2017).

Tabla2. Descripción e ítems de las escalas de Compromiso, Interés, Estrés y relación con la comunidad científica

\begin{tabular}{|c|c|c|}
\hline Escalas & Descripción & Items \\
\hline Compromi- & Grado de compromiso y & Cuando llevo a cabo mi investigación, me siento \\
\hline so & dedicación a la investiga- & lleno/a de energía \\
\hline (5 items & ción & Me siento con fuerzas para desarrollar mi proyecto \\
\hline adaptados & & de investigación \\
\hline de & & Estoy entusiasmado/a con mi trabajo de investiga- \\
\hline Pyhältö, & & ción \\
\hline \multirow[t]{3}{*}{ 2016) } & & Mi propia investigación me inspira \\
\hline & & Me siento feliz cuando empiezo a trabajar en mi \\
\hline & & investigación \\
\hline Interés & Grado de interés en la pro- & Me siento inspirado/a por mi tema de investigación \\
\hline (6 items & pia investigación & Disfruto con los retos intelectuales \\
\hline adaptados & & Quiero trabajar en la comunidad de investigadores \\
\hline de & & Quiero contribuir a mi campo de investigación \\
\hline Pyhältö, & & Me siento inspirado/a por el trabajo como investigador/a \\
\hline 2016) & & Mi investigación es útil para otros \\
\hline Estrés & Pérdida de interés sobre la & Pienso que mis estudios de doctorado son inútiles \\
\hline (11 items & propia investigación. $C y n-$ & Siento que estoy perdiendo interés en mis estudios \\
\hline adaptados & ismo & de doctorado \\
\hline de & & Me cuesta encontrar sentido a mis estudios de doc- \\
\hline Pyhältö, & & torado \\
\hline 2016) & & Antes tenía expectativas más altas sobre mis estu- \\
\hline
\end{tabular}


Carga de trabajo y responsabilidad excesivas. Agotamiento

$\begin{array}{ll}\text { Relación } & \text { Apoyo y reconocimiento } \\ \text { con la } & \text { del/los director/es } \\ \text { comuni- } & \\ \text { dad cientí- } & \\ \text { fica } & \end{array}$

(11 items adapted from Pyhältö, 2015) Apoyo y reconocimiento de otros miembros de la comunidad científica dios de doctorado de las que tengo ahora

A menudo siento que estoy fracasando en mis estudios de doctorado

A menudo me siento incompetente para realizar mis estudios de doctorado

Me siento abrumado/a por la carga de trabajo de mis estudios de doctorado

Con frecuencia duermo mal por cuestiones relacionadas con mis estudios de doctorado

Durante mi tiempo libre, doy muchas vueltas a cuestiones relacionadas con mis estudios de doctorado

La presión de mis estudios de doctorado me causa problemas en las relaciones personales

Me siento quemado/a

Recibo apoyo y atención personalizada por parte de mi(s) supervisor(es/as)

Siento que mi(s) supervisor(es/as) valora(n) mi trabajo

Me siento apreciado/a por mi(s) supervisor(es/as) Puedo discutir abiertamente cualquier problema relacionado con mi doctorado con mi(s) supervisor(es/as)

Mi experiencia es útil en la comunidad científica Siento que otros miembros de la comunidad científica valoran mi trabajo

Me siento tratado/a con respeto

Me siento aceptado/a por la comunidad científica A menudo recibo retroalimentación sobre mi trabajo en forma de crítica constructiva Hay compañerismo entre los investigadores con los que me relaciono 
Recibo apoyo y ánimos por parte de otros investigadores/as

Un equipo multicultural y multilingüe compuesto por investigadores del Reino Unido, Finlandia y España ${ }^{1}$ consensuó la adaptación al inglés de los ítems que posteriormente se tradujeron a los respectivos idiomas siguiendo un proceso de doble traducción. Todas las escalas se pilotaron con una muestra de 50 doctorandos lo que permitió ajustar su redacción y mejorar su adaptabilidad cultural.

Las variables sociodemográficas incluidas en este estudio hacían referencia al género, edad, fuente de ingresos y existencia de hijos. Además, se tuvieron en cuenta las siguientes variables académicas y profesionales (ítems de elección múltiple): disciplina, dedicación al doctorado, formato de la tesis, intención de abandono e interrupción de los estudios de doctorado, movilidad internacional, colaboración en investigación y trabajo deseado.

\section{Procedimiento}

Para la recogida de datos se contactó con las escuelas de doctorado de las universidades públicas y privadas de España que ofrecían programas de doctorado en las disciplinas indicadas en la muestra para solicitar su participación en el estudio. En aquellos casos en los que las escuelas de doctorado así lo indicaron o no respondieron se contactó con los departamentos y directores de programas.

El enlace al cuestionario estuvo abierto durante 3 meses y se enviaron -desde las escuelas de doctorado o los departamentos- tres recordatorios. La participación fue voluntaria después de recibir información sobre el proyecto a través de la misma plataforma en la que se alojó el cuestionario. El estudio contó con el visto bueno de las diferentes comisiones de ética e investigación implicadas. El Proyecto fue aprobado por el Comité de Etica de la Universitat Ramon Llull, Barcelona (CER-URL-2013_005) y por el Ministerio de Economía y Competitividad de España (CSO2013-41108-R)

\footnotetext{
${ }^{1}$ El estudio forma parte de un proyecto más amplio, sobre la formación del investigador en Ciencias Sociales y el instrumento se diseñó para recoger datos de forma paralela en los tres países mencionados. 


\section{Análisis de datos}

Para el desarrollo del estudio se adoptó un diseño observacional interpretativo de tipo transversal. La información se analizó con el programa IBM SPSS Statistics 23. Se realizaron análisis descriptivos de tendencia central y dispersión, así como ANOVA para calcular la significación de las diferencias de medias; se incluyó la d de Cohen para el tamaño del efecto. Para conocer las relaciones entre las variables se calculó la r de Pearson.

\section{Resultados}

\section{Descriptivos}

Como se puede observar en la tabla 3 y teniendo en cuenta que el punto medio de la escala es 4, la idea más compartida con respecto a la investigación es su relevancia para el investigador y para los demás (ítem 7) $(M=5.73$, d.t.=0.77). Asimismo, se considera que la investigación favorece el desarrollo personal (ítem 5) $(M=4.95$; d.t.=1.28). Con respecto a las tareas que implica investigar, los participantes son bastante conscientes de la necesidad de gestionar la información (Item 4) $(M=4.64$; d.t.=1.5). Tienen un nivel medio de consciencia sobre la necesidad de publicar artículos (item1) $(M=4.19$, d.t.=1.62) y sobre la necesidad de perseverar en el proceso (ítem 2) (ítem 6) $(M=4.19$; d.t.=1.62; $M=4.18$; d.t.=1.65) y consideran, en menor medida, que investigar supone comparar (ítem 3) $(M=4.16$; d.t.=1.51) y la investigación como vía para ser influyente y reconocimiento (ítem 8) $(M=4.10$; d.t.=1.63).

En definitiva, los participantes valoran la importancia de la investigación tanto para uno mismo y su desarrollo como para los demás. Asimismo, identifican la gestión de la información como una de las tareas más importantes en la investigación y, en menor medida, la publicación de artículos y la comparación de resultados. No se asocia fuertemente la investigación con el reconocimiento y la influencia social.

Con el fin de estudiar si las concepciones son diferentes en función de determinadas variables, se han realizado análisis de diferencia de medias, cuyos resultados se presentan a continuación.

Tabla 3. Descriptivos de las concepciones de investigación 


\begin{tabular}{lcc}
\hline Items & Media & DT \\
\hline 7.Relevante para uno mismo y los demás & 5.73 & 0.77 \\
5.Desarrollo personal & 4.95 & 1.28 \\
4.Unir, sintetizar y organizar la información & 4.64 & 1.50 \\
1.Publicar artículos y que otros los lean & 4.19 & 1.62 \\
2.Lucha, selección tema interesante para perse- & 4.19 & 1.62 \\
verar & & \\
6.Completar lo empezado & 4.18 & 1.65 \\
3.Comparar (p.e. resultados nuevos con los pre- & 4.16 & 1.51 \\
vios) & & \\
8.Ser influyente y reconocido & 4.10 & 1.63 \\
\hline
\end{tabular}

\section{Concepciones de la investigación según variables sociodemográficas}

No apareció ninguna diferencia significativa en las concepciones de la investigación en función del género. Según la edad, los mayores de $40(M=4.39$; d.t.=1.79) y los mayores de 50 años $(M=4.89$; d.t.=1.37) consideran en mayor medida que los menores de 30 años $(M=3.93$; d.t.=1.61) que la investigación supone terminar lo empezado (Item 6), siendo el tamaño del efecto pequeño $(d=0.27)$ o moderado $(d=0.64)$. También aparecen diferencias significativas en esta concepción entre los estudiantes que tienen entre 30 y 39 años $(M=4.03$; d.t=1.57) y los mayores de $50(M=4.89 ;$ d.t. $=1.37)(F(3,849)=10.376 ; p<.001)$; en este caso el tamaño del efecto es moderado $(d=-0.58)$

En función de la fuente de ingresos, los estudiantes que trabajan fuera de la universidad $(M=4.72$; d.t.=1.29) consideran en menor medida que los becarios adscritos a un proyecto $(M=5.1$; d.t.=1.19) que la investigación favorece el desarrollo personal $(F(5,854)=2.822$; $\mathrm{p}=.015)$, siendo el tamaño del efecto moderado y pequeño $(d=-0.30$ y $d=-0.15$, respectivamente). Por su parte, los que trabajan fuera de la universidad $(M=4.39$ d.t. $=1.59)$ y los becarios predoctorales $(M=4.41$; d.t.=1.67) inciden en que investigar supone terminar lo empezado en mayor medida que los becarios en un proyecto de investigación $(M=3.72 ; 1.60)$ ( $F$ (4, $855)=5.109 ; p<.001)$, teniendo en ambos casos un tamaño del efecto moderado $(d=0.42)$. 
En función de si los estudiantes tienen hijos, aquellos que sí tienen $(M=5.09$; d.t.=1.22) relacionan más la investigación con el desarrollo personal que los que no tienen hijos $(M=4.88$; d.t.=1.31) $(F(1,858)=5.461 ; p=.020)(d=0.22)$. El grupo de padres y madres $(M=4.45$; d.t.=1.61) conciben en mayor medida que los que no lo son $(M=4.04$; d.t.=1.64 $)$ que la investigación supone completar algo empezado $(F(1,858)=12.062 ; p=.001)$, tamaño del efecto $(d=0.25)$. De la misma forma, los que tienen hijos $(M=4.27$; d.t. $=1.64)$ valoran en mayor medida que los que no tienen $(M=3.99$; d.t.= 1.61) la investigación como fuente de reconocimiento e influencia $(F(1,858)=5.631 ; p=.018)$, siendo el tamaño del efecto pequeño $(d=0.17)$.

\section{Concepciones de la investigación según variables académicas y profesionales}

A continuación, se describen las diferencias en las concepciones de la investigación según variables académicas y profesionales, tales como disciplina, dedicación al doctorado, intención de abandono e interrupción de los estudios de doctorado, movilidad internacional, colaboración en investigación y trabajo deseado.

En función de la disciplina, los doctorandos de Psicología $(M=5.87$; d.t.=0.46) son más conscientes de la relevancia de la investigación para uno mismo y para los demás que los estudiantes de Ciencias Políticas y de la Información $(M=5.58$; d.t. $=0.94)[F(4,889)=3.467$; $p=.008]$, siendo el tamaño del efecto moderado $(d=0.39)$.

Con respecto al tiempo que llevan estudiando el doctorado, los que empezaron antes del 2011 consideran en mayor medida $(M=4.49$; d.t.=1.63) que los que comenzaron en 2012 $(M=3.87 ;$ dt.=1.69) que la investigación consiste en completar lo empezado $[F(8,848)$ $=3.449 ; p=.008]$; el tamaño del efecto se sitúa en $d=0.37$.

Los que tienen dedicación completa a los estudios de doctorado están menos de acuerdo $(M=4.86$; d.t.=1.28) con la potencialidad de la investigación para el desarrollo personal que los de tiempo parcial $(M=5.05 ;$ d.t. $=1.27)[F(1,889)=4.566 ; p=.033](d=0.14)$. Asimismo, los estudiantes a tiempo completo consideran en menor medida $(M=3.95$; d.t. $=1.63)$ que los que lo realizan a tiempo parcial $(M=4.43$; d.t.=1.61) que la investigación supone completar lo empezado $[F(1,858)=18.201 ; p<.001])$ con tamaño del efecto pequeño $(d=0.29)$. 
La intención de abandono de los estudios de doctorado marca diferencias en algunas concepciones de la investigación, tal y como se puede observar en la tabla 4. Los doctorandos que han tenido intención de abandonar tienen menor conciencia de que la investigación promueve el desarrollo personal, consideran en menor medida que la investigación es relevante no solo para uno mismo sino también para los demás y tienen menor motivación por ser influyentes y reconocidos por su investigación. Consideran en menor medida que investigar suponer comparar informaciones.

Tabla 4. Diferencias significativas en concepciones de investigación según intención de abandono de estudios de doctorado. Grupo 1 Intención de abandono / Grupo 2 no intención de abandono

\begin{tabular}{llllc}
\hline Concepción & F (sig.) & Grupo (M; d.t) & d Cohen \\
\hline $\begin{array}{l}\text { 3.Comparar (ej. resultados } \\
\text { nuevos con previos) }\end{array}$ & $4,054(.044)$ & G1 $(4 ; 1.50)$ & G2 $(4.22 ; 1.52)$ & -0.14 \\
$\begin{array}{l}\text { 5. Desarrollo personal } \\
\text { 7,424 (.007) }\end{array}$ & G1 (4.77; 1.30) & G2 $(5.03 ; 1.27)$ & -0.20 \\
$\begin{array}{l}\text { 7.Relevante para uno mis- } \\
\text { mo y los demás }\end{array}$ & 7,615(.006) & G1 (5.64; 0.88) & G2 (5.79;0.68) & -0.19 \\
$\begin{array}{l}\text { 8. Ser influyente y recono- } \\
\text { cido }\end{array}$ & $6,328(.012)$ & G1 (3.90; 1.64) & G2 (4.20; 1.60) & -0.18 \\
\hline
\end{tabular}

Los estudiantes que en algún momento han interrumpido sus estudios de doctorado $(M=4.48$; d.t.=1.58) son más conscientes que los estudiantes que han tenido continuidad $(M=4.11 ;$ d.t. $=1.65)$ de que investigar suponer completar lo empezado $(F(1,883)=6.649$; $p=.010)$, con tamaño del efecto pequeño $(d=0.22)$. El haber realizado alguna estancia o visita de investigación al extranjero no marca diferencias significativas en las concepciones sobre la investigación. Tampoco aparecen diferencias significativas en las concepciones según las condiciones de la actividad doctoral, individual, mixta o en equipo. 
Los únicos aspectos vinculados a la colaboración en investigación que marcan diferencias en las concepciones tienen que ver con la participación en congresos nacionales e internacionales. Así, los estudiantes que no han presentado ninguna comunicación en congresos nacionales tienen una conciencia menor de que investigar supone publicar artículos para que otros los lean $(M=3.92$; d.t.=1.718) que los estudiantes que han presentado entre una y dos comunicaciones $(M=4.42$; d.t.=1.47) y entre tres y cuatro $(M=4.44$; d.t.=1.52) ( $F$ (4, 889)=5.234; $p=.000$ ). El tamaño del efecto de las diferencias entre ambos grupos y los estudiantes que no han presentado ninguna comunicación es moderado ( $d=-0.31$ y $d=-0.32)$.

Por su parte, los estudiantes que han presentado una o dos comunicaciones en congresos nacionales son más conscientes de que investigar supone unir, sintetizar y organizar la información $(M=4.88$; d.t.=1.37) que los que han presentado entre tres y cuatro $(M=4.42$; d.t.=1.58) $(F(4,889)=3.410 ; p=.009)(d=0.31)$.

Los doctorandos que han presentado más de siete comunicaciones en congresos internacionales consideran la investigación como vía de reconocimiento y de influencia social $(M=4.69 ;$ d.t.=1.41) en mayor medida que los que han presentado 1 o $2(M=4.01 ;$ d.t.=1.64) $(\mathrm{F}$ $(4,889)=2.959 ; p=.019)$. El tamaño del efecto es moderado $(d=0.44)$. Los estudiantes que tienen alguna publicación consideran que investigar consiste en publicar artículos $(M=4.31$; d.t.=1.58) y que otros los lean en mayor medida que los doctorandos que no han publicado $(M=3.98$; d.t.=1.66 $)(F(1,892)=8.4407 ; p=.004)$; entre ambos grupos el tamaño del efecto es pequeño $(d=0.20)$.

La tabla 5 incluye las diferencias significativas en las concepciones de investigación según trabajo deseado. En esta variable se han distinguido cinco grupos de expectativas: G1 exclusivamente docencia, G2 Exclusivamente investigación, G3 ambos pero principalmente docencia, G4 ambos pero principalmente investigación, G5 sin interés en desarrollar una carrera académica. Aquellos que no tienen interés en seguir la carrera académica no asumen tanto como los que aspiran a dedicarse tanto a la docencia como a la investigación la relevancia que ésta tiene para uno mismo y los demás. Por su parte, aquellos que quieren dedicarse exclusivamente a la investigación valoran en menor medida que los que quieren dedicarse tanto a la docencia como a la investigación, la posibilidad que da la investigación para ser influyente y reconocido. 
Tabla 5. Diferencias significativas en concepciones de investigación según trabajo deseado

\begin{tabular}{lcccc}
\hline Concepción & F & \multicolumn{2}{c}{ Grupo $(\mathrm{M} ;$ d.t $)$} & d Cohen \\
\hline 7. Relevante para uno & $5.773^{* * *}$ & G5 $(5.46 ; 1.18)$ & G3 $(5.74 ; 0.75)$ & 0.28 \\
mismo y los demás & & G4 $(5.85 ; 0.47)$ & 0.43 \\
& & & \\
8. Ser influyente y & 4.262** & G2 $(3.15 ; 1.65)$ & G3 $(4.23 ; 1.60)$ & 0.66 \\
reconocido & & G4 $(4.14 ; 1.56)$ & 0.61
\end{tabular}

Nota: $* * \mathrm{p}<.01 ; * * * \mathrm{p}<.001$

\section{Relación entre concepciones de la investigación y otras variables}

El interés general por el doctorado tiene una relación significativa positiva y baja con la concepción de la investigación como relevante, como vía de reconocimiento y de desarrollo personal. Con el resto de concepciones tiene una relación significativa positiva y muy baja. Especificando según las dimensiones de interés por la investigación (ver tabla 6), se constata que el interés enfocado en la contribución a la comunidad y a los retos tiene una correlación positiva baja con la concepción de la investigación como medio para el desarrollo personal, o como vía de reconocimiento, y como algo relevante para uno mismo y los demás, con el resto tiene una correlación positiva pero muy baja.

El interés del doctorado centrado en la mejora de la propia situación laboral tiene una relación significativa muy baja con casi todas las concepciones de investigación, excepto con la investigación como gestión de la información, con la que no tiene relación significativa y con la concepción de la investigación como vía de influencia y reconocimiento, con la que mantiene una relación significativa baja. El interés del doctorado enfocado en el desarrollo personal tiene una relación significativa con todas las concepciones de la investigación. La mayoría de ellas son relaciones positivas muy bajas, excepto las concepciones relacionadas con el desarrollo personal, la relevancia para uno mismo y los demás, y el reconocimiento personal, con las que la relación es baja.

Tabla 6. Correlaciones r entre concepciones de investigación e interés por la investigación $(N=894)$ 


\begin{tabular}{|c|c|c|c|c|}
\hline Concepciones/Interés & Interés $\mathrm{T}$ & Comunidad & Posición & Desarrollo \\
\hline 1.Publicar artículos y que otros los lean & $.181 * * *$ & $.122 * * *$ & $.179 * * *$ & $.137 * * *$ \\
\hline $\begin{array}{l}\text { 2.Lucha, selección tema interesante } \\
\text { para perseverar }\end{array}$ & $.134 * * *$ & $.079 * *$ & $.126 * * *$ & $.127 * * *$ \\
\hline $\begin{array}{l}\text { 3.Comparar (p.e. resultados nuevos con } \\
\text { los previos) }\end{array}$ & $.126 * * *$ & $.099 * *$ & $.103 * *$ & $.121 * * *$ \\
\hline $\begin{array}{l}\text { 4.Unir, sintetizar y organizar la infor- } \\
\text { mación }\end{array}$ & $.100 * *$ & $.077 *$ & .073 & $.133 * * *$ \\
\hline 5.Desarrollo personal & $.229 * * *$ & $.242 * * *$ & $.107^{* *}$ & $.295 * * *$ \\
\hline 6.Completar lo empezado & $.166^{* * * *}$ & $.140 * * *$ & $.109^{* *}$ & $.196^{* * *}$ \\
\hline 7.Relevante para uno y demás & $.282 * * *$ & $.358 * * *$ & $.126^{* * *}$ & $.300 * * *$ \\
\hline 8. Ser influyente y reconocido & $.268 * * *$ & $.173 * * *$ & $.259 * * *$ & $.224 * * *$ \\
\hline
\end{tabular}

Nota: $* p<.05 ; * * p<.01 ; * * * p<.001$

En la tabla 7 se pueden observar las correlaciones entre las concepciones de la investigación y el estrés asociado a la formación investigadora, tanto general como en las dos dimensiones, pérdida de interés y sobrecarga de trabajo y responsabilidad. Todas las correlaciones significativas son muy bajas. Se podría afirmar que hay una mínima relación entre ambas dimensiones de estrés y el considerar la investigación como una lucha en la que hay que perseverar. La pérdida de interés se asocia negativamente en un nivel muy pequeño con la consideración de la investigación como relevante.

Tabla 7. Correlaciones $r$ entre concepciones de investigación y estrés $(N=889)$

\begin{tabular}{|c|c|c|c|}
\hline Concepciones/Otras variables & $\begin{array}{c}\text { Tensión } \\
\text { total }\end{array}$ & $\begin{array}{l}\text { Pérdida } \\
\text { interés }\end{array}$ & Sobrecarga \\
\hline 1.Publicar artículos y que otros los lean & .061 & .032 & $.073^{*}$ \\
\hline $\begin{array}{l}\text { 2.Lucha, selección tema interesante para perseve- } \\
\text { rar }\end{array}$ & $.142 * * *$ & $.108 * *$ & $.135 * * *$ \\
\hline $\begin{array}{l}\text { 3.Comparar (p.e. resultados nuevos con los pre- } \\
\text { vios) }\end{array}$ & .012 & .003 & .018 \\
\hline 4.Unir, sintetizar y organizar la información & $.075^{*}$ & $.067 *$ & .061 \\
\hline 5.Desarrollo personal & .021 & $-.097 *$ & $.068^{*}$ \\
\hline
\end{tabular}


6.Completar lo empezado

7.Relevante para uno y demás

8.Ser influyente y reconocido
.064

.065

.059
.019

$-.134 * * *$

.019
.093

$.086 * *$

Nota: $* p<.05 ; * * p<.01 ; * * * p<.001$

La relación entre el apoyo percibido por la dirección y la comunidad científica y las concepciones de la investigación se presentan en la tabla 8. Se da una correlación significativa positiva y baja entre el apoyo percibido por el director o directora de la tesis y la consideración de la investigación como relevante para uno mismo y los demás y muy baja y positiva con la concepción de la investigación como vía de desarrollo personal. El apoyo percibido por parte de la comunidad tiene relaciones significativas muy bajas con la mayoría de las concepciones de investigación, excepto con la idea de investigación como lucha y como completar lo empezado.

Tabla 8. Correlaciones entre concepciones de investigación y apoyo $(N=894)$

\begin{tabular}{lccc}
\hline Concepciones/Otras variables & Apoyo total & Dirección & Comunidad \\
\hline 1.Publicar artículos y que otros los lean & $.113^{* *}$ & .063 & $.145^{* * *}$ \\
2.Lucha, selección tema interesante para perse- & .024 & .006 & .040 \\
verar & & & \\
3.Comparar (p.e. resultados nuevos con los & $.089^{* *}$ & .051 & $.112^{* *}$ \\
previos) & & & \\
4.Unir, sintetizar y organizar la información & $.075^{*}$ & .039 & $.100^{* *}$ \\
5.Desarrollo personal & $.186^{* * *}$ & $.165^{* * *}$ & $.169^{* * *}$ \\
6.Completar lo empezado & .044 & .024 & .058 \\
7.Relevante para uno y demás & $.324^{* * *}$ & $.289^{* * *}$ & $.293^{* * *}$ \\
8.Ser influyente y reconocido & $.106^{* *}$ & .063 & $.132^{* * *}$ \\
\hline
\end{tabular}

Nota: $* p<.05 ; * * p<.01 ; * * * p<.001$

En la Tabla 9 se recogen las correlaciones entre las concepciones de la investigación y el grado de compromiso con la investigación doctoral. 
Tabla 9. Correlaciones r entre concepciones de investigación y compromiso

\begin{tabular}{lc}
\hline Concepciones/Otras variables & Compromiso \\
\hline 1.Publicar artículos y que otros los lean & .039 \\
2.Lucha, selección tema interesante para perseverar & .008 \\
3.Comparar (p.e. resultados nuevos con los previos) & .049 \\
4.Unir, sintetizar y organizar la información & .039 \\
5.Desarrollo personal & $.179^{* * *}$ \\
6.Completar lo empezado & .056 \\
7.Relevante para uno y demás & $.265^{* * *}$ \\
8.Ser influyente y reconocido & $.083^{* *}$ \\
\hline
\end{tabular}

Note: $* * p<.01 ; * * * p<.001$

El compromiso con la investigación doctoral tiene una relación significativa positiva y baja con la concepción de que la investigación es relevante para uno mismo y los demás y una relación muy baja con la concepción de la investigación como un medio de desarrollo personal y de reconocimiento. Por el contrario, el compromiso con la investigación doctoral no guarda relación significativa con las restantes concepciones que consideran investigar como ser influyente y reconocido; unir, sintetizar y organizar la información; publicar artículos y que otros los lean, lucha, selección de un tema interesante para perseverar, o como completar lo empezado y comparar resultados.

\section{Discusión y Conclusiones}

En este estudio se planteó conocer las concepciones sobre la investigación de los estudiantes de doctorado en Ciencias Sociales en el contexto español y analizar su relación con algunas variables relevantes en la formación investigadora. Este análisis ha mostrado que los doctorandos entienden la investigación prioritariamente como un proceso orientado tanto a ofrecer una contribución a la comunidad como al desarrollo personal. Se percibe también, aunque en menor medida, como un producto que se aporta a la comunidad organizando la información de forma coherente para que tenga sentido y que se plasma en la publicación de artículos (Akerlind, 2008; Brew, 2001; Meyer et al. 2005; 2007; Stubbs et al. 2014). Sin em- 
bargo, no vinculan de una manera importante la investigación con el reconocimiento y la influencia social (Äkerlind, 2008).

Investigadoras e investigadores no se diferencian en sus concepciones de la investigación, pero sí los grupos de edad. Las diferencias aumentan con la edad y se perciben diferencias, posiblemente generacionales, por ejemplo, en la concepción de que la investigación supone terminar lo empezado. Igualmente, la fuente de ingresos y tener o no hijos, circunstancias vitales destacadas en estudios previos por su influencia en el desarrollo del proceso doctoral (Coromina et al., 2011), se asocia con diferentes modos de entender qué es investigar.

Los becarios adscritos a un proyecto y los doctorandos que tienen hijos relacionan la investigación con el desarrollo personal más que el resto. Ambos colectivos, junto con los que trabajan fuera de la universidad manifestaron que investigar supone completar lo empezado y, además, aquellos que tienen hijos consideraron que la investigación es fuente de reconocimiento e influencia. Las dificultades actuales para acceder y desarrollar una carrera académica son objeto de preocupación supranacional, tal y como queda patente en el estudio de Hauss, Kaulisch y Tesch (2015) sobre la carrera académica en el caso de los doctorandos alemanes, o en el de Turner y McAlpine (2011) sobre académicos y doctorandos en Reino Unido, y probablemente resultan claves para entender estas concepciones. Por otra parte, los que desearían dedicarse a la docencia y a la investigación consideran que investigar les permitirá ser influyentes y reconocidos. La disonancia entre estas expectativas y las dificultades existentes debería ser tenida en cuenta en la formación y en la dirección doctoral.

Estas concepciones también presentaron diferencias significativas, aunque bajas en función de variables académicas. En concreto, en relación con la disciplina los doctorandos de Psicología son más conscientes de la relevancia de la investigación para uno mismo y para los demás que los de Ciencias Políticas y de la información, un resultado similar al que encontraron Stubb, Pyhältö y Lonka (2014) con doctorandos de ciencias del comportamiento frente a los de salud o ciencias naturales. La fecha de inicio del doctorado es una variable relevante porque está relacionada con planes de estudio diferentes. Los que empezaron antes del 2011 consideraron en mayor medida que los que comenzaron después que la investigación consiste en completar lo empezado. Esta fecha coincide con la extinción de planes antiguos, en los que no se limitaba la duración y la entrada en vigor de un nuevo plan (RD 99/2011) en el que se 
incorpora esta limitación. La duración media de los doctorandos de CCSS en planes antiguos era de nueve años (de Miguel, et. al., 2004). Aquellos que tienen dedicación a tiempo parcial frente a los de dedicación completa a los estudios destacaron la potencialidad de la investigación para el desarrollo personal y la necesidad de que la investigación implique completar lo empezado. Gran parte de este grupo tiene alguna ocupación profesional fuera del ámbito académico o, en menor medida, responsabilidades familiares. Parece que al aumentar la duración de los estudios la necesidad de completar lo empezado se destaca explícitamente.

Una valoración menor de la investigación para el desarrollo personal, de su relevancia para sí mismo y para los demás, para ser influyentes y reconocidos puede afectar a la persistencia en el proceso doctoral. Por su parte, quienes en algún momento han interrumpido sus estudios fueron más conscientes de que investigar supone completar lo empezado, es decir, quizá esta concepción les ha ayudado a retomar los estudios.

En los programas de doctorado actuales, las estancias internacionales, la participación en congresos y las publicaciones se han incorporado como recomendaciones o incluso como requisitos. Asimismo, la vinculación a un equipo de investigación durante todo el proceso se ha convertido en una práctica cada vez más habitual. Los resultados sugieren que la participación en congresos y la publicación marcan diferencias significativas en creencias relacionadas con comunicar los resultados, elaborar conocimiento y ser reconocido socialmente. Estas parecen sugerir que la propia práctica fortalece la concepción lo que merecería su consideración para diseñar la formación investigadora. Sin embargo, haber realizado alguna estancia en el extranjero y llevar a cabo la actividad doctoral de manera individual, mixta o en equipo no se tradujeron en diferencias significativas en las concepciones. La importancia atribuida a ambas circunstancias dentro de la formación doctoral en el contexto internacional indica la necesidad de seguir investigando al respecto. Así, sería relevante clarificar el concepto de comunidad científica, así como sus modalidades (híbridas, estructuradas, uni o interdisciplinares...) y su influencia en las concepciones.

\section{Limitaciones y prospectivas}

Este estudio proporciona una valiosa información sobre las concepciones de los investigadores, pero su carácter trasversal la limita a un momento de la formación investigadora. Para entender cómo evolucionan estas concepciones e inciden en el proceso de formación de 
la identidad del investigador sería interesante realizar un estudio longitudinal que permitiera comparar cómo evolucionan las concepciones en diferentes etapas del proceso doctoral y su incidencia en la formación de la identidad del investigador.

Por otra parte, aporta un instrumento útil para conocer las concepciones de los investigadores en formación pero convendría avanzar en la consolidación de instrumentos fiables que permitan confirmar la organización de las concepciones en las dimensiones, desarrollo individual- desarrollo de la comunidad y proceso-producto, identificadas en este estudio, empezando por un análisis confirmatorio de la estructura de este instrumento. Además, un estudio cualitativo permitiría conocer en profundidad qué percepciones tienen los doctorandos sobre lo que significa investigar y la vivencia que tienen de los eventos significativos vinculados a dichas percepciones.

\section{Agradecimientos}

Este estudio forma parte del proyecto de I+D+i Orientada a los Retos de la Sociedad "Formación de la identidad del Investigador Novel en Ciencias Sociales (FINS)" y fue financiado por el Ministerio de Economía y Competitividad [CSO2013-41108-R].

\section{Referencias}

Åkerlind, G.S. (2008). An academic perspective on research and being a researcher: An Integration of the literature. Studies in Higher Education 33(1), 17-31. doi:10.1080/03075070701794775.

Badía, A., Meneses, J. y Monereo, C. (2014). Affective Dimension of University Professors about their Teaching: An Exploration through the Semantic Differential Technique. Universitas Psychologica 13(1). doi:10.11144/Javeriana.UPSY13-1.adup.

Baker, V. L. y Lattuca, L. R. (2010). Developmental networks and learning: toward an interdisciplinary perspective on identity development during doctoral study. Studies in Higher Education, 35(7), 807-827. doi:10.1080/03075070903501887.

Brew, A. (2001). Conceptions of research: A phenomenographic study. Studies in Higher Education 26, (3), 271-285. doi 10.1080/03075070120076255.

Buela-Casal, G., Bermudez, M., Sierra, J., Ramiro, A., Castro, G., Paz Bermudez, M. y Teresa Ramiro, J. (2011). Performance analysis of doctoral studies in accordance with the 
regulations of doctoral degree in Spanish universities. Cultura \& Educacion, 23(2), 285-296. doi:10.1174/113564011795944767.

Castelló, M. González, L. \& Iñesta, A. (2010). La regulación de la escritura académica en el doctorado: El impacto de la revisión colaborativa en los textos. Revista Española de Pedagogía, 247, 521-537.

Castelló, M., Iñesta, A. \& Monereo, C. (2009). Towards self-regulated academic writing: an exploratory study with graduate students in a situated learning environment. Electronic Journal of Research in Educational Psychology, 7(3), 1107-1130. Disponible en: http://www.investigacionpsicopedagogica.org/revista/new/english/ContadorArticulo.php?367

Castelló, M., Iñesta, A., \& Corcelles, M. (2013). Learning to Write a Research Article: Ph. D. Students' Transitions toward Disciplinary Writing Regulation. Research in the Teaching of English, 47(4), 442.

Castelló, M., McAlpine, L. \& Pyhältö, K. (2017). Spanish and UK post-PhD researchers: Writing perceptions, well-being and productivity. Journal of Higher Education Research \& Development, 1-15. http://dx.doi.org/10.1080/07294360.2017.1296412

Castelló, M., Pardo, M., Sala-Bubaré, A. \& Suñe, N. (2017). Why do students consider to drop out of doctoral degrees? Institutional and personal factors. Higher Education, 116, doi:10.1007/s10734-016-0106-9

Coromina, Ll., Capó, A., Guia, J. y Coenders,G. (2011). Effect of Background, Attitudinal and Social Network Variables on PhD Students' Academic Performance. A Multimethod Approach. Estudios sobre Educación, 20, 233-253.

Chitez, M. y Kruse, O. (2012). Writing cultures and genres in European higher education. In M. Castelló y C. Donahue (Eds.), University writing: Selves and texts in academic societies (pp. 151-175). Bingley, UK: Emerald Group Publishing Limited.

Hauss, K., Kaulisch, M. y Tesch, T., (2015). Against all odds: determinants of doctoral candidates' intention to enter academia in Germany, International Journal for Researcher. Development, 6(2), 122-143. doi:.1108/IJRD-07-2014-0015.

McAlpine, L., Amundsen, C. y Turner, G. (2013). Constructing post-PhD careers: Negotiating opportunities and personal goals. International Journal for Researcher Development, 4(1), 39-54. doi:10.1108/IJRD-01-2013-0002. 
McAlpine, L., Amundsen, C. y Turner, G. (2014). Identity-trajectory: Reframing early career academic experience. British Educational Research Journal, 40(6), 952-969. doi:10.1002/berj.3123.

McAlpine, L., Jazvac-Martek, M. y Hopwood, N. (2009). Doctoral student experience in Education: Activities and difficulties influencing identity development. International Journal for Researcher Development, 1(1), 97-109. doi:10.1108/1759751X201100007.

McAlpine, L. y McKinnon, M. (2013). Supervision-the most variable of variables: Student perspectives. Studies in Continuing Education, 35(3), 265-280. doi:10.1080/0158037X.2012.746227.

Mainhard, T., Van der Rijst, R., Van Tartwijk, J. y Wubbels, T. (2009). A model for the supervisor-doctoral student relationship. Higher Education, 58(3), 359-373. doi:10.1007/s10734-009-9199-8.

Martinsuo, M. y Turkulainen, V. (2011). Personal commitment, support and progress in doctoral studies. Studies In Higher Education, 36(1), 103-120. doi:10.1080/03075070903469598.

Meyer, J.H.F., Shanahan, M.P. y Laugksch, R.C. (2005). Students' conceptions of research I: A qualitative and quantitative analysis. Scandinavian Journal of Educational Research 49(3), 225-44. doi:10.1080/00313830500109535.

Meyer, J. H., Shanahan, M. P. y Laugksch, R. C. (2007). Students' conceptions of research. 2: An exploration of contrasting patterns of variation. Scandinavian Journal of Educational Research, 51(4), 415-433. doi:10.1080/00313830701485627.

de Miguel, J.M., Sarabia Heydrich, B., Vaquera, E;G. y Amirah, H. (2004). ¿Sobran o faltan doctores?. Empiria. Revista de Metodología de Ciencias Sociales, 7, 162-155. Disponible en http://search.proquest.com/docview/1312683055? accountid=14529.

Musi-Lechuga, B., Olivas-Ávila, J. A. y Castro Vázquez, A. (2011). Productividad de los programas de doctorado en psicología con mención de calidad en artículos de revistas incluidas en el Journal Citation Reports. Psicothema, 23(3), 343-348. Disponible en http://www.psicothema.com/psicothema.asp?id=3892.

Pitcher, R. (2010). The Self in Research and Other Matters: A Study of Doctoral Students' Conceptions. International Journal for Researcher Development, Vol. 1 Iss 3 pp. 248 256 Permanent link to this document: doi:10.1108/1759751X201100018. 
Pitcher, R. (2011). Doctoral students' conceptions of research. The Qualitative Report, 16(4), 971-983. Disponible en http://www.nova.edu/ssss/QR/QR16-4/pitcher.pdf.

Pitcher, R. y Åkerlind, G.S. (2009). Post-doctoral researchers' conceptions of research: A metaphor analysis. Retrieved from Canberra, Australia The Australian National University, The Centre for Educational Development and Academic Methods: http://www.dspace.cam.ac.uk/bitstream/1810/224927/1/3513.pdf.

Pyhältö, K. M., Peltonen, J., Rautio, P., Haverinen, K., Laatikainen, M. y Vekkaila, J. E. (2016). Summary report on doctoral experience in UniOGS graduate school at the University of Oulu. Acta Universitatis Ouluensis. http://jultika.oulu.fi/Record/isbn978952-62-1084-1.

Pyhältö, K., Stubb, J. and Lonka, K. (2009). Developing scholarly communities as learning environments for doctoral students, International Journal for Academic Development, 14(3), 221-232. doi:10.1080 / 13601440903106551.

Pyhältö, K., Vekkaila, J. y Keskinen, J., (2015). Fit matters in the supervisory relationship: Doctoral students' and supervisors' perceptions about supervisory activities. Innovations in Education and Teaching International. 52, 1, 4-16. doi:10.1080/14703297.2014.981836.

Stubb, J., Pyhältö, K. y Lonka, K. (2012). The Experienced Meaning of Working with a PhD Thesis. Scandinavian Journal of Educational Research, 56(4), 439-456. doi: 10.1080/00313831.2011.599422.

Stubb, J., Pyhältö, K. y Lonka, K., (2014) Conceptions of research: the doctoral student experience in three domains. Studies in Higher Education, 39(2), 251-264, doi:10.1080/03075079.2011.651449.

Turner, G. y McAlpine, L. (2011). Doctoral experience as researcher preparation: activities, passion, status. International Journal for Researcher Development, 2(1), 46-60. doi: $10.1108 / 17597511111178014$.

Vekkaila, J., Pyhältö, K. y Lonka, K. (2014). Engaging and disengaging doctoral experiences in the behavioural sciences. International Journal for Researcher Development, 5(1), 33-55. doi:10.1108/IJRD-09-2013-0015.

Villardón-Gallego, L. y Yániz, C. (2013). Propuesta de un Plan de Tutoría y Apoyo a estudiantes de doctorado. Revista de Docencia Universitaria. REDU. 11(2), Mayo-Agosto, 135-152. Recuperado de http://red-u.net 
Visser-Wijnveen, G. J., Van Driel, J.H., Van der Rijst, R. M., Verloop, N., \& Visser, A. (2009) The relationship between academics' conceptions of knowledge, research and teaching - a metaphor study, Teaching in Higher Education, 14(6), 673-686, doi:10.1080/13562510903315340. 